\title{
Duas Formas Alternativas da Equação de um Balanço ENTÁLPICO A UM REACTOR
}

ISABEL M. A. FonsecA

A realização de balanços energéticos, em particular, em sistemas com reaç̧ão química, apresenta uma maior complexidade do que os balanços mássicos. Neste artigo deduzem-se duas formas alternativas da equação dum balanço entálpico a um reactor. A resolução de um exemplo, recorrendo às duas equações, permite avaliar a utilidade de cada uma delas e o grau de dificuldade que Ihes está inerente.

\section{INTRODUÇ̃̃o}

Lecciono, há já alguns anos, uma disciplina - Estequiometria Industrial - cujo tronco principal é a realização de balanços mássicos e energéticos em sistemas em estado estacionário. A disciplina dirige-se a alunos das licenciaturas de Química Industrial e Engenharia Química.

Os balanços, equações que resultam da aplicação dos princípios da conservação da massa e da energia a um determinado sistema, constituem uma ferramenta essencial em domínios tão importantes como operações unitárias, cinética, dinâmica de sistemas e outros. Em tom de brincadeira, costumo dizer aos alunos que toda a gente faz balanços, não dá é por isso. E, para sustentar a minha afirmação, refiro como exemplo, os balanços de "massa" (em euros) que todos realizamos quando pagamos uma despesa no hipermercado - o que entra na caixa registadora menos o que sai (troco) dá o que se acumula. Na sequência deste "balanço", introduzo a equação geral dum balanço a um determinado sistema na forma que considero mais pedagógica:

$\left(\begin{array}{c}\text { velocidade de entrada } \\ \text { de matéria (ou energia) } \\ \text { no sistema }\end{array}\right]-\left(\begin{array}{c}\text { velocidade de saída } \\ \text { de matéria (ou energia) } \\ \text { no sistema }\end{array}\right]=\left(\begin{array}{c}\text { velocidade de acumulação } \\ \text { de matéria (ou energia) } \\ \text { no sistema }\end{array}\right]$

Por conseguinte, o balanço de massa e o balanço de energia apresentam a mesma forma. Se o termo da acumulação for nulo, diz-se que o sistema

\footnotetext{
Departamento de Engenharia Química da Universidade de Coimbra Pinhal de Marrocos, Pólo II Coimbra, Portugal fonseca@eq.uc.pt
}

está em estado estacionário, o que significa que o conteúdo do sistema (em matéria ou energia) não se altera com o tempo. Nestas circunstâncias a eq. (1) pode ser escrita na forma, no caso dum balanço de energia. As quantidades de energia indicadas reportam-se a um certo intervalo de tempo.

A minha experiência, com amostras significativas de alunos ( 120 alunos), veio demonstrar que eles compreendem e realizam mais facilmente os balanços de matéria, do que os balanços de energia. A quantificação da massa de um sistema, ou dos caudais que entram e saem do sistema, que é necessário fazer para efectuar o balanço mássico é relativamente intuitiva. O mesmo não se verifica com a energia, que é um conceito mais complexo. Um balanço de energia tem de incluir vários termos diferentes que traduzem as várias formas em que a energia pode existir (energia interna, potencial, cinética) e os diferen-

es mecanismos de transferência de energia (por transferência de massa, por transferência de calor ou por realização de trabalho). Outra razão pela qual os balanços energéticos são mais complexos do que os mássicos, prende-se com o facto de os valores das energias internas e das entalpias utili$\left[\begin{array}{c}\text { quantidade de energia } \\ \text { que entra no sistema }\end{array}\right]-\left[\begin{array}{c}\text { quantidade de energia } \\ \text { que entra no sistema }\end{array}\right]=0$

zadas nos balanços serem referidos a um determinado estado de referência que se admite ter energia interna ou entalpia nulas. Embora a escolha do estado de referência seja, em princí-

pio, arbitrária, existem estados mais adequados do que outros, o que se traduzirá por uma simplificação da equação do balanço. Convém aqui relembrar que a definição do estado de referência implica a especificação da temperatura, pressão e estado de agregação. Por simplificação, este último é algumas vezes omitido, no caso de todas as espécies se encontrarem no mesmo estado. Os valores da energia interna e da entalpia relativas (definidas em relação a um estado de referência) encontram-se tabelados. Por exemplo, os valores destas grandezas para a água encontram-se nas "Steam Tables", onde o estado de referência adoptado é a água pura, no seu ponto triplo, ao qual se atribui o valor zero.

Os diversos livros de texto que abordam esta questão apresentam metodologias diferentes para a realização dos balanços energéticos, umas mais pedagógicas do que outras. Considero que aquela que refiro aqui é das mais simples e facilmente assimilável pelos alunos.

Na maioria dos processos, os termos do balanço relativos às energias cinética e potencial apresentam uma grandeza desprezável quando comparados com os termos da entalpia, do 
calor e do trabalho e, portanto, para fins práticos, não são considerados. $O$ balanço energético é então designado por balanço entálpico.

Considere-se o sistema seguinte, com apenas uma corrente de entrada (e), e uma corrente de saída (s), por uma questão de simplificação, em estado estacionário:

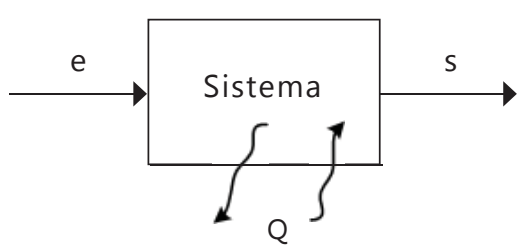

Figura 1 Sistema com uma corrente de entrada (e) e uma corrente de saída (s) em estado estacionário. Q representa a quantidade de calor transferida entre o sistema e o seu exterior por unidade de tempo

O balanço entálpico a este sistema é, de acordo com a eq. (2):

$$
\left(H^{e}+Q\right)-\left(H^{s}\right)=0
$$

ou

$$
\mathrm{H}^{\mathrm{e}}+\mathrm{Q}=\mathrm{H}^{\mathrm{s}}
$$

em que $\mathrm{H}^{\mathrm{e}}$ e $\mathrm{H}^{\mathrm{s}}$ representam as entalpias das correntes de entrada e de saída do sistema, respectivamente, e $Q$ a quantidade de calor transferida entre o sistema e o exterior (por convenção, positiva se é transferida para dentro do sistema, e negativa se é para fora). Estas três quantidades são referidas a um intervalo de tempo. A eq. (4) representa um balanço entálpico a um sistema aberto em estado estacionário, referido a um certo intervalo de tempo.

A importância da função entalpia $(\mathrm{H}=\mathrm{U}+\mathrm{PV})$ advém do facto de considerar implicitamente o trabalho do fluxo efectuado pelo fluido em movimento nas tubagens das correntes de entrada e saída do sistema, questão que é abordada em detalhe em vários livros de texto [1-3]. As entalpias $\mathrm{H}^{\mathrm{e}}$ e $\mathrm{H}^{\mathrm{s}}$ contidas na eq. (4) são valores absolutos. No entanto, os valores da entalpia que se encontram tabelados são valores relativos, ou seja, estão referidos a um estado de referência ao qual se atribui entalpia nula. A relação entre a entalpia absoluta, $\mathrm{H}$, e a entalpia relativa, $\mathrm{H}^{r}$, é dada pela expressão,
$\mathrm{H}=\mathrm{H}^{\mathrm{r}}+\mathrm{H}^{0}$

sendo $\mathrm{H}^{0}$ a entalpia do estado de referência. Asubstituição das entalpias absolutas na eq. (4), conduz à expressão:

$\left(H^{e, r}+H^{0}\right)+Q=\left(H^{s, r}+H^{0}\right)$

que devido ao cancelamento dos termos da entalpia do estado de referência origina,

$H^{e, r}+Q=H^{s, r}$

equação formalmente análoga à eq. (4).

Em conclusão, os balanços de energia podem ser escritos em termos das entalpias absolutas ou relativas. $\mathrm{Na}$ prática, não dispomos de entalpias absolutas e, por isso, usam-se sempre entalpias relativas. Contudo, há que ter particular cuidado para que os valores da entalpia nos balanços se reportem ao mesmo estado de referência.

Vamos abrir aqui um parêntesis para introduzir alguns conceitos que são essenciais para realizar balanços entálpicos a sistemas onde ocorre reacção química. Começaremos por referir velocidade de reacção, $r$, pela expressão,

$r=\frac{\mathrm{N}_{\mathrm{i}}^{\mathrm{s}}-\mathrm{N}_{\mathrm{i}}^{\mathrm{e}}}{v}$,

em que $\mathrm{N}_{i}^{\mathrm{s}}$ e $\mathrm{N}_{i}$ e representam as quantidades do componente $\mathrm{i}$, na maioria das vezes expressas em moles, à saída e à entrada do reactor, respectivamente, durante um intervalo de tempo. $v$ é o coeficiente estequiométrico da espécie i na reacção acertada. Convencionou-se que este coeficiente é positivo se a espécie for um produto, e negativo se for um reagente. A velocidade exprime-se em (moles. tempo ${ }^{-1}$ ), e é independente da espécie i escolhida para o seu cálculo.

Outro parâmetro importante é a entalpia de reacção que se define pela expressão,

$$
\begin{aligned}
& \Delta \mathrm{H}_{\mathrm{R}}(\mathrm{T}, \mathrm{P})=\sum_{\text {produtosi }} \mathrm{n}_{\mathrm{i}} \mathrm{H}_{\mathrm{i}}(\mathrm{T}, \mathrm{P})- \\
& -\sum_{\text {reagentes }} \mathrm{n}_{\mathrm{j}} \mathrm{H}_{\mathrm{j}}(\mathrm{T}, \mathrm{P})
\end{aligned}
$$

que nos indica que é a diferença en- tre a entalpia dos produtos (i) e a dos reagentes (j) nas condições $(T, P) . H_{i}$ $(T, P)$ representa a entalpia da espécie i e $n_{i}$ o respectivo coeficiente estequiométrico.

Esta expressão pode ser apresentada na forma mais sintética

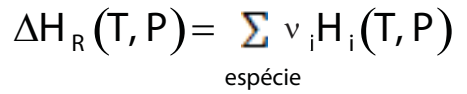

desde que se adopte a convenção anteriormente definida para o sinal do coeficiente estequiométrico $v$.

O cálculo da entalpia da reacção em condições padrão (1atm, 298K) ${ }^{1}$ pode ser efectuado a partir das entalpias de formação, $\Delta \mathrm{H}_{\mathrm{f}, \mathrm{i}}^{0}$, das espécies intervenientes na reacção pela expressão,

$$
\Delta \mathrm{H}_{\mathrm{R}}^{0}=\sum_{\mathrm{i}} v_{\mathrm{i}} \Delta \mathrm{H}_{\mathrm{f}, \mathrm{i}}^{0}
$$

A entalpia de formação da espécie i é a entalpia da reacção de formação desta espécie a partir dos elementos que a constituem, em condições padrão. Pode ser definida por,

$$
\Delta \mathrm{H}_{\mathrm{f}, \mathrm{i}}^{0}=\mathrm{H}_{\mathrm{i}}^{0}-\sum_{\mathrm{e}} \alpha_{\mathrm{e}, \mathrm{i}} \mathrm{H}_{\mathrm{e}}^{0}
$$

onde tal como anteriormente, $\mathrm{H}_{\mathrm{i}}^{0}$ é a entalpia padrão da espécie i. $\mathrm{H}_{\mathrm{e}}^{0}$ é a entalpia padrão do elemento e que possui um coeficiente atómico, $\alpha_{e, j}$, na espécie i. O somatório é efectuado sobre todos os elementos que constituem a espécie.

\section{BALANÇO DE ENERGIA A UM SISTEMA COM REACÇÃO QUÍMICA}

\section{EQUAÇÃo DO BALANÇO QUE EXPLICITA A ENTALPIA DA REACÇÃO}

Vamos então considerar a figura que se segue (fig. 2), que consiste num reactor em estado estacionário, com apenas uma corrente de entrada e uma corrente de saída, onde ocorre uma única reacção química. Os componentes das duas correntes encontram-se no mesmo estado de agregação, por uma questão de simplificação. 


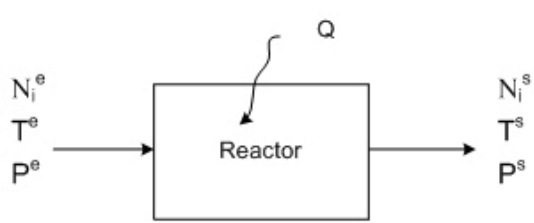

Figura 2 Reactor com uma corrente de entrada (e) e uma corrente de saída (s). T e P representam respectivamente a temperatura e pressão das correntes e $\mathrm{N}_{i}$ a quantidade do componente i presente na corrente num certo intervalo de tempo, $\mathrm{Q}$ é a quantidade de calor transferida num certo intervalo de tempo

Admitindo que as correntes são misturas ideais, e considerando desprezáveis todas as outras energias, e que o sistema não realiza trabalho, podemos escrever o balanço entálpico ao reactor na forma:

$$
\sum \mathrm{N}_{\mathrm{i}}^{\mathrm{e}} \mathrm{H}_{\mathrm{i}}^{\mathrm{e}}+\mathrm{Q}=\sum \mathrm{N}_{\mathrm{i}}^{\mathrm{s}} \mathrm{H}_{\mathrm{i}}^{\mathrm{s}}
$$

em que $\mathrm{H}_{i}{ }^{e}$ e $\mathrm{H}_{i}^{\mathrm{s}}$ representam as entalpias específicas da espécie i $\left(\mathrm{Jmol}^{-1}\right)$, respectivamente à entrada e à saída do reactor. Vamos supor que seleccionamos para estado de referência de cada espécie, $\mathrm{H}_{\mathrm{i}}^{0}$, à temperatura $\mathrm{T}^{0} \mathrm{e}$ pressão $\mathrm{P}^{0}$. Se ao membro esquerdo da eq. (13) adicionarmos os seguintes termos [2]:

$$
\left(\sum \mathrm{N}_{\mathrm{i}}^{\mathrm{s}} \mathrm{H}_{\mathrm{i}}^{0}-\sum \mathrm{N}_{\mathrm{i}}^{\mathrm{s}} \mathrm{H}_{\mathrm{i}}^{0}\right)
$$

e

$$
\left(\sum \mathrm{N}_{\mathrm{i}} \mathrm{H}_{\mathrm{i}}^{0}-\sum \mathrm{N}_{\mathrm{i}}^{\mathrm{e}} \mathrm{H}_{\mathrm{i}}^{0}\right)
$$

e agruparmos os termos associados à corrente de entrada e à corrente de saída, o balanço adquire a forma

$$
\begin{aligned}
& \sum \mathrm{N}_{\mathrm{i}}^{\mathrm{e}}\left(\mathrm{H}_{\mathrm{i}}^{\mathrm{e}}-\mathrm{H}_{\mathrm{i}}^{0}\right)+\mathrm{Q}- \\
& -\sum_{\mathrm{i}} \mathrm{H}_{\mathrm{i}}^{0}\left(\mathrm{~N}_{\mathrm{i}}^{\mathrm{s}}-\mathrm{N}_{\mathrm{i}}^{\mathrm{e}}\right)=\sum \mathrm{N}_{\mathrm{i}}^{\mathrm{s}}\left(\mathrm{H}_{\mathrm{i}}^{\mathrm{s}}-\mathrm{H}_{\mathrm{i}}^{0}\right)
\end{aligned}
$$

Pela definição de velocidade de reacção, eq. (8), $\left(\mathrm{N}_{i}{ }^{\mathrm{s}}-\mathrm{N}_{i}{ }^{\mathrm{e}}\right)=v \mathrm{r}$, e tendo em conta a definição de entalpia de reacção, eq. (10), podemos concluir que

$$
\sum H_{i}^{0}\left(N_{i}^{s}-N_{i}^{e}\right)=r \Delta H_{R}^{0}
$$

Substituindo a eq. (15) na expressão (14) obtemos:

$$
\begin{aligned}
& \sum N_{i}^{e} H_{i}^{e, r}+r\left(-\Delta H_{R}^{0}\right)+Q=. \\
= & \sum N_{i}^{s} H_{i}^{s, r}
\end{aligned}
$$

em que

$$
\begin{aligned}
& \mathrm{H}_{\mathrm{i}}^{\mathrm{e}, \mathrm{r}}=\left(\mathrm{H}_{\mathrm{i}}^{\mathrm{e}}-\mathrm{H}_{\mathrm{i}}^{0}\right) \\
& \mathrm{e} \\
& \mathrm{H}_{\mathrm{i}}^{\mathrm{s}, \mathrm{r}}=\left(\mathrm{H}_{\mathrm{i}}^{\mathrm{s}}-\mathrm{H}_{\mathrm{i}}^{0}\right)
\end{aligned}
$$

representam as entalpias específicas relativas ao estado de referência, da espécie i. Ou apresentando ainda a eq. (16) numa forma mais sintética, e facilmente memorizável [4],

$$
H^{e}+\mathrm{Q}+\mathrm{r}\left(-\Delta \mathrm{H}_{\mathrm{R}}^{0}\right)=\mathrm{H}^{\mathrm{s}}
$$

Omitimos o superescrito $r$ para simplificar.

É contudo conveniente não esquecer o significado dos vários termos da eq. (17). Assim,

- $\mathrm{H}^{\mathrm{e}}$ e $\mathrm{H}^{\mathrm{s}}$ representam as entalpias relativas das correntes de entrada e de saída, respectivamente, por unidade de tempo. Cada uma é um somatório das entalpias de cada espécie presente na corrente, à qual se atribuiu o estado de referência $\left(\mathrm{T}^{0}, \mathrm{P}^{0}\right)$;

- Q é a quantidade de calor transferida num certo intervalo de tempo. Obedece à convenção de sinais;

- r é a velocidade da reacção;

- $\Delta \mathrm{H}_{\mathrm{R}}{ }^{0}$ representa a entalpia da reacção nas condições $\left(\mathrm{T}^{0}, \mathrm{P}^{0}\right)$. Obedece à convenção de sinais.

\section{Equação do Balanço Que Não Expl-} cita o Termo de Entalpia da Reacção

A eq. (17) contém individualizado o termo da entalpia da reacção, que para a maioria das reacções pode ser determinada a partir das entalpias de formação padrão que se encontram tabeladas para muitos compostos, o que a torna particularmente útil.

Vamos agora referir uma forma alternativa da equação do balanço de energia que não incorpora explicitamente o termo da entalpia de reacção.

Considere-se novamente a eq. (14),

$$
\begin{aligned}
& \sum \mathrm{N}_{\mathrm{i}}^{\mathrm{e}}\left(\mathrm{H}_{\mathrm{i}}^{\mathrm{e}}-\mathrm{H}_{\mathrm{i}}^{0}\right)+\mathrm{Q}- \\
- & \sum \mathrm{H}_{\mathrm{i}}^{0}\left(\mathrm{~N}_{\mathrm{i}}^{\mathrm{s}}-\mathrm{N}_{\mathrm{i}}^{\mathrm{e}}\right)=\sum \mathrm{N}_{\mathrm{i}}^{\mathrm{s}}\left(\mathrm{H}_{\mathrm{i}}^{\mathrm{s}}-\mathrm{H}_{\mathrm{i}}^{0}\right)
\end{aligned}
$$

e em vez de substituirmos o termo $\left(\mathrm{N}_{\mathrm{i}}^{\mathrm{s}}\right.$ $\mathrm{N}_{\mathrm{i}} \mathrm{e}$ ), usemos a definição de entalpia de formação (eq. (12)) e explicitemos $\mathrm{H}_{\mathrm{i}}^{0}$

$$
\mathrm{H}_{\mathrm{i}}^{0}=\Delta \mathrm{H}_{\mathrm{f}, \mathrm{i}}^{0}+\sum \alpha_{\mathrm{e}, \mathrm{i}} \mathrm{H}_{\mathrm{e}}^{0}
$$

O $3^{\circ}$ termo do $1^{\circ}$ membro da eq. (14) pode ser alterado, substituindo nele esta última expressão (18):

$$
\sum \mathrm{H}_{\mathrm{i}}^{0}\left(\mathrm{~N}_{\mathrm{i}}^{\mathrm{s}}-\mathrm{N}_{\mathrm{i}}^{\mathrm{e}}\right)=\sum \mathrm{H}_{\mathrm{i}}^{0} \mathrm{~N}_{\mathrm{i}}^{\mathrm{s}}-\sum \mathrm{H}_{\mathrm{i}}^{0} \mathrm{~N}_{\mathrm{i}}^{\mathrm{e}}(
$$

$=\sum \mathrm{N}_{\mathrm{i}}^{\mathrm{s}}\left(\Delta \mathrm{H}_{\mathrm{f}, \mathrm{i}}^{0}+\sum_{\mathrm{e}} \alpha_{e, i} \mathrm{H}_{\mathrm{e}}^{0}\right)-\sum \mathrm{N}_{\mathrm{i}}^{\mathrm{e}}\left(\Delta \mathrm{H}_{\mathrm{f}, \mathrm{i}}^{0}+\sum_{\mathrm{e}} \alpha_{\mathrm{e}, \mathrm{i}} \mathrm{H}_{\mathrm{e}}^{0}\right)$

$$
=\sum \mathrm{N}_{\mathrm{i}}^{s} \Delta \mathrm{H}_{\mathrm{f}, \mathrm{i}}^{0}-\sum \mathrm{N}_{\mathrm{i}}^{\mathrm{e}} \Delta \mathrm{H}_{\mathrm{f}, \mathrm{i}}^{0}+\sum_{\mathrm{e}} \mathrm{H}_{\mathrm{e}}^{0}\left[\sum_{\mathrm{i}}^{\alpha}{ }_{\mathrm{e}, \mathrm{i}}\left(\mathrm{N}_{\mathrm{i}}^{\mathrm{s}}-\mathrm{N}_{\mathrm{i}}^{\mathrm{e}}\right) \mid\right.
$$

O termo $\sum \alpha_{e, i}\left(N_{i}^{s}-N_{i}^{e}\right)=0$, uma vez que se trata do conjunto dos balanços mássicos aos e elementos, $\mathrm{e}=1, \ldots, \mathrm{e}$. Então, substituindo (21) em (14) e rearranjando a equação obtém-se

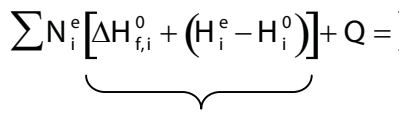

(a)

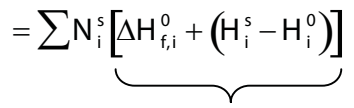

(b)

Os termos (a) e (b) são constituídos pela entalpia de formação da espécie i mais a entalpia dessa espécie em relação ao estado padrão (298 $\mathrm{K}, 1 \mathrm{~atm})$. Se não há reacção, então $\mathrm{N}_{\mathrm{i}}^{\mathrm{e}}=\mathrm{N}_{\mathrm{i}}^{\mathrm{s}}$, e portanto os termos $\Delta \mathrm{H}_{\mathrm{f}, \mathrm{i}}^{0}$ cancelam-se. Contudo, se houver reacção, então $\mathrm{N}_{\mathrm{i}}^{\mathrm{e}} \neq \mathrm{N}_{\mathrm{i}}^{\mathrm{s}}$ e os termos $\Delta \mathrm{H}_{\mathrm{f}, \mathrm{i}}^{0}$ darão conta implicitamente da entalpia da reacção. Deste modo é possível definir a entalpia total de uma corrente j pela expressão

$H^{j}=\sum N_{i}^{j}\left[\Delta H_{f, i}^{0}+\left(H_{i}^{j}-H_{i}^{0}\right)\right](23)$

Então o balanço, eq. (22), reduz-se à forma simples,

$\mathrm{H}^{\mathrm{e}}+\mathrm{Q}=\mathrm{H}^{\mathrm{s}}$

Esta forma de equação do balanço é particularmente adequada para implementar em computador uma vez que não é necessário efectuar o cálculo separado da entalpia da reacção. 
Apresenta-se a seguir um problema resolvido pelas duas formas do balanço entálpico, equações (17) e (24) [1], onde é possível comparar os dois processos de cálculo.

Exemplo: O óxido nítrico pode ser obtido a partir da oxidação parcial do $\mathrm{NH}_{3}$ gasoso, com ar. No reactor dá entrada uma corrente gasosa de $\mathrm{NH}_{3}$, a $25^{\circ} \mathrm{C}$, e uma corrente de ar a $750^{\circ} \mathrm{C}$, ambas a $1 \mathrm{~atm}$. A conversão de $\mathrm{NH}_{3}$ é $90 \%$. Sabendo que a temperatura da corrente que sai do reactor é de $920^{\circ} \mathrm{C}$, calcule a quantidade de calor que é necessário retirar do reactor por mole de $\mathrm{NH}_{3}$ alimentado. Considere que por cada mole de $\mathrm{NH}_{3}$ que entra no reactor entram 2,4 moles de $\mathrm{O}_{2}$.

\section{Dados:}

$4 \mathrm{NH}_{3}(\mathrm{~g})+5 \mathrm{O}_{2}(\mathrm{~g}) \rightarrow 4 \mathrm{NO}(\mathrm{g})+6 \mathrm{H}_{2} \mathrm{O}(\mathrm{g})$

$\Delta \mathrm{H}_{\mathrm{R}}\left(920^{\circ} \mathrm{C}, 1 \mathrm{~atm}\right)=-216,42 \mathrm{kcal} \mathrm{mol}^{-1}$

$\Delta \mathrm{H}_{\mathrm{f}, \mathrm{NH}_{3}(\mathrm{~g})}^{0}=-10,92 \mathrm{kcal} \mathrm{mol}^{-1}$

$\Delta \mathrm{H}_{\mathrm{f}, \mathrm{H}_{2} \mathrm{O}(\mathrm{g})}^{0}=-57,8 \mathrm{kcal} \mathrm{mol}^{-1}$

$\Delta \mathrm{H}_{\mathrm{f}, \mathrm{NO}(\mathrm{g})}^{0}=21,6 \mathrm{kcal} \mathrm{mol}^{-1}$

As capacidades caloríficas dos gases na forma $c_{p}=a+b T+c^{2}+d^{3}$, podem ser retiradas do apêndice 3 da referência [1].

Considere a figura abaixo que representa as várias correntes do reactor.

Base de cálculo: $1 \mathrm{~mol}$ de $\mathrm{NH}_{3}$ na corrente 2 por hora

A velocidade da reacção será então,

$\mathrm{r}=\frac{0,9}{4}=0,225 \mathrm{~mol}_{\mathrm{h}} \mathrm{h}^{-1}$

Se escolhermos para temperatura de referência, $\mathrm{T}^{0}=920^{\circ} \mathrm{C}(1193 \mathrm{~K})$, então a entalpia da corrente de saída é nula, $\mathrm{H}^{3}=0$, e a equação do balanço energético, eq. (17), reduz-se a:

$$
\begin{gathered}
\mathrm{H}^{1}+\mathrm{H}^{2}+\mathrm{Q}+\mathrm{r}\left(-\Delta \mathrm{H}_{\mathrm{R}}\right)=0 \\
\text { ou } \\
\mathrm{H}^{1}+\mathrm{H}^{2}+\mathrm{r}\left(-\Delta \mathrm{H}_{\mathrm{R}}\right)=-\mathrm{Q}
\end{gathered}
$$

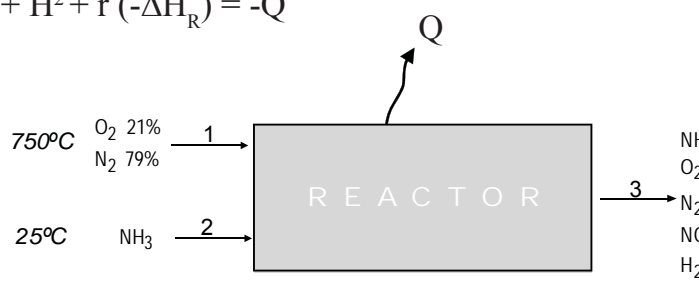

$$
\begin{aligned}
\mathrm{N}_{\mathrm{O}_{2}}^{1} & =2,4 \mathrm{~mol}^{-1} \\
\mathrm{~N}_{\mathrm{N}_{2}}^{1} & =2,4 \times \frac{0,79}{0,21} \\
& =9,03 \mathrm{~mol}^{-1}{ }^{-1}
\end{aligned}
$$

Substituindo na equação anterior do balanço, tem-se:

$$
\begin{gathered}
2,4 \int_{1193}^{1023} c_{p \mathrm{O}_{2}} d T+9,03 \int_{1193}^{1023} c_{p N_{2}} d T+ \\
+0,225(-216420)+\int_{1193}^{298} c_{p N H_{3}} d T=-Q \\
Q=-22730 \text { cal. } \mathrm{h}^{-1} \\
\mathrm{Q}=-22,73 \mathrm{kcal} . \mathrm{h}^{-1}
\end{gathered}
$$

Vamos agora resolver o problema por aplicação da equação geral do balanço de energia, eq. (24):

$$
\mathrm{H}^{1}+\mathrm{H}^{2}+\mathrm{Q}=\mathrm{H}^{3}
$$

sendo a entalpia de cada corrente obtida pela expressão,

$$
\mathrm{H}^{\mathrm{j}}=\sum \mathrm{N}_{\mathrm{i}}^{\mathrm{j}}\left(\Delta \mathrm{H}_{\mathrm{f}, \mathrm{i}}^{0}+\int_{\mathrm{T}^{0}}^{\mathrm{T}^{\mathrm{j}}} \mathrm{c}_{\mathrm{p}_{\mathrm{i}}} \mathrm{dT}\right)
$$

equivalente à eq. (23). $c_{p_{i}}$ é a capacidade calorífica da espécie i no intervalo de temperatura $\left[\mathrm{T}^{0}, \mathrm{~T}^{\mathrm{T}}\right]$.

Assim, a entalpia da corrente 1 será dada por:

$$
\begin{aligned}
\mathrm{H}^{1} & =2,4\left(0+\int_{298}^{1023} \mathrm{c}_{\mathrm{pO}_{2}} \mathrm{dT}\right)+9,03\left(0+\int_{298}^{1023} \mathrm{c}_{\mathrm{pN}_{2}} \mathrm{dT}\right) \\
& =61716 \mathrm{cal} . \mathrm{h}^{-1}
\end{aligned}
$$

Para a corrente 2 tem-se:

$$
\begin{aligned}
\mathrm{H}^{2} & =1\left(-10920+\int_{298}^{298} \mathrm{c}_{\mathrm{pNH}_{3}} \mathrm{dT}\right) \\
& =-10920 \mathrm{cal} \cdot \mathrm{h}^{-1}
\end{aligned}
$$

Efectuando os balanços mássicos, tendo em atenção a conversão do $\mathrm{NH}_{3}$ podemos determinar a composição da corrente de saída, 3 .

$$
\left\{\begin{array}{l}
N_{N_{3}}^{3}=0,1 \\
N_{N O}^{3}=0,9 \\
N_{\mathrm{O}_{2}}^{3}=1,275 \\
N_{N_{2}}^{3}=9,03 \\
N_{\mathrm{H}_{2} \mathrm{O}}^{3}=1,35
\end{array}\right.
$$

A entalpia da corrente 3 será então dada pela expressão:

$$
\begin{aligned}
& \mathrm{H}^{3}=0,1\left(-10920+\int_{298}^{1193} \mathrm{c}_{\mathrm{pNH}_{3}} \mathrm{dT}\right)+ \\
& +0,9\left(21600+\int_{298}^{1193} \mathrm{c}_{\mathrm{pNO}} \mathrm{dT}\right)+ \\
& +1,275\left(0+\int_{298}^{1193} \mathrm{c}_{\mathrm{p}_{2}} \mathrm{dT}\right)+9,03\left(0+\int_{298}^{1193} \mathrm{c}_{\mathrm{p}_{2}} \mathrm{dT}\right)+ \\
& +1,35\left(-57800+\int_{298}^{1193} \mathrm{c}_{\mathrm{p}_{2} \mathrm{O}} \mathrm{dT}\right)= \\
& =-41,6+25728+9085+60280-66980 \\
& =28071 \mathrm{cal} . \mathrm{h}^{-1}
\end{aligned}
$$

Substituindo na equação do balanço vem:

$$
\begin{aligned}
61716-10920+\mathrm{Q} & =28071 \\
\mathrm{Q} & =-22725 \mathrm{cal} . \mathrm{h}^{-1} \\
& =-22,73 \mathrm{kcal} . \mathrm{h}^{-1}
\end{aligned}
$$

A equação do balanço de energia geral é mais complicada quando os cálculos são efectuados manualmente, devido ao facto de se escolher geralmente para estado de referência o estado padrão $\left(25^{\circ} \mathrm{C}\right.$, 1atm). Esta escolha implica que se retenham todos os termos do balanço. No entanto, esta forma é muito útil nas situações em que a estequiometria da reacção é mais complexa, ou quando os caudais das espécies têm que ser determinados por balanços aos elementos.

\section{Conclusão}

A realização de balanços energéticos, em particular em sistemas com reacção química, reveste-se de maior complexidade do que a realização dos balanços mássicos. Partindo da equação geral dum balanço de energia, apresentam-se duas equações alternativas dum balanço entálpico 
a um sistema onde ocorre reacção química: uma equação onde o termo da entalpia da reacção aparece individualizado, e outra onde não aparece individualizado, sendo as entalpias de formação das espécies os termos que implicitamente dão conta da entalpia da reacção. A primeira forma é particularmente útil quando se dispõe do valor da entalpia da reacção à temperatura de uma ou mais correntes. A escolha desta temperatura como referência implica a anulação das entalpias dessas correntes, o que se traduz por uma simplificação dos cálculos, aspec- to muito importante nas resoluções manuais. A segunda forma envolve mais cálculos, sendo particularmente útil para implementar no computador e nas situações em que a estequiometria da reacção é mais complexa (ou desconhecida) e ainda quando os caudais das espécies são determinados por balanços aos elementos.

\section{NotA}

${ }^{1}$ Actualmente considera-se que a pressão do estado de referência é 1 bar. Contudo, em muitas tabelas de dados termodinâmicos estes ainda se referem à pressão de $1 \mathrm{~atm}$, sendo por isso ainda usada em alternativa.

\section{REFERÊNCIAS}

[1] G. V. Reklaitis, Introduction to Material and Energy Balances, 1st edition, John Wiley \& Sons, USA, 1983.

[2] R. M. Murphy, Introduction to Chemical Processes, - Principles, Analysis, Synthesis, McGraw - Hill edition, 2007.

[3] R. M. Felder, R. W. Rousseau, Elementary Principles of Chemical Processes, 2nd edition, John Wiley \& Sons, Canada, 1986.

[4] I. M. A.Fonseca, A. P. V. Egas, Fundamentos de Balanços de Energia, Cadernos de Engenharia Química $n^{\circ} 7$, Universidade de Coimbra, 2002.

\section{Actualidade Científica}

\section{Partindo Ligações com Efeito DE Estufa}

Os fluorocarbonetos são compostos que encontram muitas utilizações especialmente depois de o protocolo de Montreal ter banido a utilização de clorofluorocarbonetos (CFCs) por provocarem a destruição da camada de ozono.

Para além de muito utilizados como fluidos de refrigeração em frigoríficos e unidades de ar condicionado, as propriedades químicas da ligação C-F tornam os fluorocarbonetos ideais para utilizações que exijam materiais resistentes quimica e termicamente.

São ainda muito hidrofóbicos, pelo que podem ser utilizados como revestimento em materiais à prova de água, não aderentes ou repelentes de sujidade, encontrando aplicações desde a óptica ao vestuário passando por utensílios de cozinha.

Para além disso, a elevada solubilidade do oxigénio nestes compostos faz com que sejam um componente base do sangue artificial.

Os fluorocarbonetos, FCs, são igualmente gases de efeito de estufa (GEEs) muito potentes, com o problema adicional de a sua inércia química e a sua resistência térmica os tornarem persistentes no meio ambiente.
Por exemplo, o tetrafluorometano, análogo fluorado do metano, persiste na atmosfera por 50000 anos.

As razões pelas quais os FCs são tão atractivos para inúmeras aplicações estão igualmente na origem da enorme dificuldade em os tratar, já que a quebra da ligação C-F só pode ser conduzida a temperaturas muito elevadas o que limita a adopção de processos que destruam estes compostos.

Assim, muitos países comprometeram-se pelo protocolo de Kyoto a diminuir significativamente as emissões de FCs em 2012

A importância dos FCs no mundo actual, por um lado, e por outro os seus problemas ambientais explicam o interesse despertado por um artigo publicado por um grupo da universidade Brandeis na Science de 29 de Agosto. O grupo de Oleg Ozerov sintetizou um catalisador que parte ligações C-F à temperatura ambiente, prometendo para breve uma arma química eficaz na guerra a estes compostos.
Os produtos da reacção apresentada são hidrocarbonetos e fluorosilanos que não apresentam os problemas dos compostos de partida. Os autores conseguiram neutralizar todo 0 material à temperatura ambiente em três FCs testados (num dos casos em apenas 6 horas).

Alguns especialistas da área comentaram que há alguns problemas a resolver antes de o processo poder ser utilizado em larga escala, nomeadamente em relação à síntese e reactividade do catalisador.

Mas, como declarou Ozerov ao Enviromental Research Web, «Em termos de relevância ambiental, é possível que esta investigação abra as portas para potenciais novas tecnologias que removam os poluentes ambientais fluorados. Eu devo frisar que a investigação tal como está neste momento não é prática, mas o artigo da Science é uma prova de princípio, não é uma tentativa de demonstrar que pode ser aplicada já.»

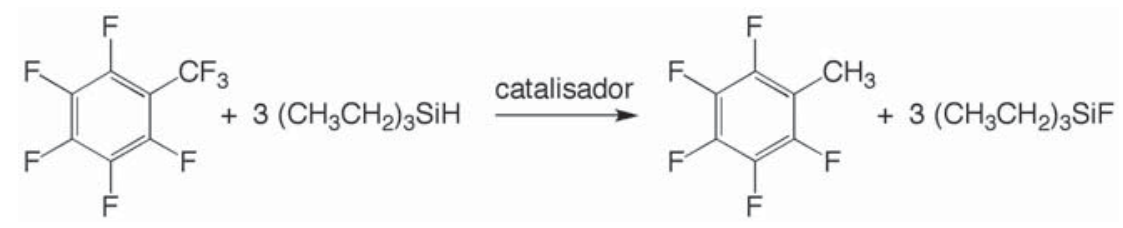




\section{Actualidade Científica}

\section{Química e ARTE}

No número de Julho da revista Analytical Chemistry o artigo «Visualization of a Lost Painting by Vincent van Gogh Using Synchrotron Radiation Based X-ray Fluorescence Elemental Mapping» descreve como uma equipa multidisciplinar envolvendo a Universidade Técnica de Delft, a Universidade de Antuérpia, o museu Kro"ller-Müller, o Centro de Investigação e Restauração dos Museus Franceses e dois aceleradores de partículas, o DESY em Hamburgo e o ESRF (Instalação Europeia de Radiação Sincrotrónica) em Grenoble, revelou o rosto de uma camponesa que durante 121 anos permaneceu escondido sob um «Pedaço de relva».

Van Gogh reciclava as suas telas pintando sobre elas obras diferentes - os especialistas consideram que até um terço das primeiras obras do artista ocultam outras composições. Investi- gações preliminares tinham revelado que esse era o caso do quadro «Patch of Grass», pintado em Paris em 1887 e exposto no museu Kro"ller-Müller, na cidade holandesa de Otterlo.

As técnicas convencionais de raios- $X$ utilizadas neste tipo de análise apenas permitiam ver sob as camadas de pintura mais superficiais vagos traços de uma cabeça, que se pensa poder fazer de uma série pintada por Van Gogh entre 1884-85, durante a estadia na aldeia holandesa de Nuenen em que pintou "Os comedores de batatas», considerado o seu primeiro grande trabalho.

Os cientistas resolveram então examinar pela primeira vez um quadro com radiação sincrotrão. Em Hamburgo, o quadro foi analisado por fluorescência de raios- $X$, técnica que permitiu revelar os pigmentos utilizados nas várias camadas de tinta e criar um modelo a três dimensões do esboço. As camadas superficiais mostraram ser constituidas principalmente por tintas incorporando sais de zinco, bário e enxofre depositadas sobre uma camada uniforme de um sal de chumbo, que foi usado como um primário que escondeu a pintura anterior e preparou a tela para uma nova. Para esboçar a cabeça da camponesa, van Gogh utilizou cinabre, sulfureto de mercúrio utilizado durante milénios como o pigmento vermelho de eleição e para iluminar determinadas zonas da face, van Gogh recorreu ao amarelo de Nápoles ou amarelo de antimónio, $\mathrm{Pb}\left(\mathrm{SbO}_{3}\right)_{2} / \mathrm{Pb}_{3}\left(\mathrm{Sb}_{3} \mathrm{O}_{4}\right)_{2}$, misturado com branco de zinco. A fluorescência do antimónio e do mercúrio permitiu recriar a cores e com uma precisão sem precedentes o esboço escondido.

\section{Actualidade Científica}

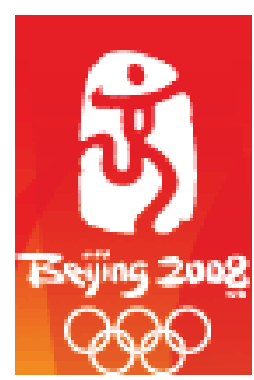

\section{Nanologo Olímpico}

A equipa de Chad A. Mirkin, professor da Universidade de Northwestern (EUA) e director do Instituto Internacional de Nanotecnologia de Northwestern, produziu em massa o logotipo dos Jogos Olímpicos de Pequim, com dimensões micrométricas: 15000 logotipos numa área de 1 centímetro quadrado (2500 logotipos caberiam num grão de arroz). Para definir o logotipo, os pontos chegaram a ter 90 nanómetros de diâmetro.

A técnica de impressão usada (Polymer Pen Lithography) utiliza filas de canetas fabricadas em polímero, permitindo imprimir em grandes áreas a três escalas diferentes: nanométrica, micrométrica e milimétrica.

Para aplicar este método é necessário utilizar um Microscópio de Força Atómica (AFM).

Refira-se que C.A. Mirkin desenvolveu uma outra técnica de padronização à escala nanométrica, designada por Dip Pen Lithography que também usa um AFM, usando a ponta de prova do AFM como caneta que, mergulhada numa tinta, permite transferir esse material (DNA ou materiais semicondutores) para um substrato criando estruturas com resolução nanométrica (Adaptado de Small Times, 15 de Agosto). 